\title{
Molecular marker assisted screening for kernel polyphenol oxidase activity in wheat Triticum aestivum L.
}

\author{
Abhishek Prasad \\ Department of Plant Breeding and Genetics, Bihar Agricultural College, Bihar Agricultural \\ University, Sabour, Bhagalpur (Bihar)-813210, India \\ Present address: National Institute of Plant Genome Research, Aruna Asaf Ali Marg, New \\ Delhi-110067, India \\ Tirthartha Chattopadhyay* \\ Department of Plant Breeding and Genetics, Bihar Agricultural College, Bihar Agricultural \\ University, Sabour, Bhagalpur (Bihar)-813210, India \\ *Corresponding author. Email: tirthartha@gmail.com
}

\section{How to Cite}

Prasad, A. and Chattopadhyay, T. (2021). Molecular marker assisted screening for kernel polyphenol oxidase activity in wheat Triticum aestivum L.. Journal of Applied and Natural Science, 13(1), 8 - 15. https://doi.org/10.31018/jans.v13i1.2445

\begin{abstract}
High level of kernel polyphenol oxidase (PPO) activity has been found to be the major factor behind time-dependent discolouration of wheat-based products, which ultimately leads to reduced consumers' preference. Till date, 6 genes belonging in 2 paralogous sets present in wheat (Triticum aestivum L.) chromosome 2 homeologues (2A, 2B and 2D) have been reported to govern kernel PPO activity. Among these 6 genes, perfect molecular markers have been developed for 2 genes (PPOA1 and PPOD1) and the major role of PPOA1 gene in governing kernel PPO activity in wheat has been reported. In the present study we have used the molecular markers for the PPOA1 and PPOD1 genes to characterize wheat genotypes for their kernel PPO activity. We have successfully converted the dominant marker assay for the PPOD1 locus into a co-dominant assay using the already reported primers. Our molecular screening strategy could explain the kernel PPO activity of wheat genotypes in rapid, reliable and environment-independent manner. Furthermore, biochemical estimation of kernel PPO activity in wheat genotypes indicated the involvement of other genes in fine-tuning this important trait. Thus, the present study should facilitate the breeders in marker-assisted selection and breeding for developing wheat genotypes with low kernel PPO activity.
\end{abstract}

Keywords: Co-dominant screening; Marker-assisted breeding; Paralogous genes; Phenol test; Wheat kernel polyphenol oxidase activity

\section{INTRODUCTION}

Time-dependent darkening of wheat-based products, particularly noodles, is undesirable from the point of view of consumers' preference. The ubiquitous enzyme polyphenol oxidase (PPO, EC 1.14.18.1) has been reported to be the major factor behind this discolouration of different wheat-based products (Baik et al., 1995; Mares and Campbell, 2001; Fuerst et al., 2006). The nuclear-encoded, copper-containing, oxygendependent PPO enzyme has been reported to possess monophenolase and diphenolase activities (Gao et al., 2009). Thus, in presence of oxygen, PPO is involved in hydroxylation of $o$-monophenols to o-diphenols and subsequently $o$--diphenols to o-quinones. The resulting quinones produce dark brown or black colouration after reacting with amines and thiol groups or through non-enzymatic self-polymerization (Mayer and Harel, 1979; Baik et al., 1994; Anderson and Morris, 2001). In wheat kernel, PPO activity is mainly localized in the bran layer, which is generally removed during milling. But contaminating residual bran layer has been reported to be sufficient for causing product discolouration (Hatcher and Kruger, 1993; Rani et al., 2001). In contrast, the nutritive value of wheat bran (Stevenson et al., 2012) has been realized by the consumers to shift their choice from refined wheat flour to whole grain wheat flour, to a great extent. Furthermore, flour protein content has been reported to have negative correlation with PPO activity (Park et al., 1997). In this way, breeding for development of wheat genotypes with low kernel PPO 
activity is rewarding, not only from the point of view of consumers' preference but also for the nutritional enrichment of wheat-based products.

The genetics behind kernel PPO activity in wheat has been studied in great details, where different $P P O$ genes of wheat has been classified in 'kernel' and ' non-kernel' clusters (Jukanti et al., 2004; Anderson et al., 2006). The major genes governing kernel PPO activity have been reported to be present in wheat homeologous group 2 chromosomes (Jime'nez and Dubcovsky, 1999). The role of the PPO genes on chromosome 2AL and 2DL (i.e., PPOA1 and PPOD1) in regulating kernel PPO activity has been established (Sun et al., 2005; Chang et al., 2007; He et al., 2007; Wang et al., 2009) and the PPOA1 locus has been found to have major effect in determining kernel PPO activity (Raman et al., 2007; Beecher and Skinner, 2011; Martin et al., 2011; Nilthong et al., 2013). In order to facilitate marker assisted selection (MAS), reproducible gene-based sequence tag site (STS) markers have been developed for the PPOA1 and PPOD1 loci. However, the possibility of erroneous prediction of alleles at PPOD1 locus using the developed markers has been indicated (Nilthong et al., 2013). In the recent past, 4 QTLs for PPO activity on chromosomes 2AL, 3BS, 4BL and 7DL has been documented to explain 64.1-89.0\% of the total phenotypic variance across environments (Zhai et al., 2016). Application of diversity arrays technology (DArT) in doubled haploid population has yielded markers (wPt-7024, wPt-0094 and wPt-2544) associated with major loci governing kernel PPO activity in bread wheat (Sadeque et al., 2018). Recently, genome-wide association study has identified several QTLs that regulate kernel PPO activity ; out of these QTLs, 12 located on homoeologous group 1 chromosomes, chromosomes $4 \mathrm{~B}, 4 \mathrm{D}$, and $7 \mathrm{~A}$ and $7 \mathrm{~B}$ have been postulated as new PPO loci (Zhai et al., 2020). In the present study, we analyzed the allelic variation at PPOA1 and PPOD1 loci in 14 wheat genotypes and attempted to correlate their allelic status with kernel PPO activity. Using PPO33 marker for the PPOA1 locus, we document the identification of PPOA1b low PPO allele in selected wheat genotypes. At the same time, we propose the strategy of using 3 already reported primers (for PPO16 and PPO29 markers) in a single polymerase chain reaction (PCR) for codominant screening of the PPOD1 locus to identify the PPOD1a low PPO allele.

\section{MATERIALS AND METHODS}

\section{Seed materials}

Freshly harvested seeds (kernels) of 14 wheat (Triticum aestivum L.) genotypes were obtained from Wheat Section, Bihar Agricultural University, Sabour, Bhagalpur.

\section{Bioinformatics analysis}

Sequence information of the wheat polyphenol oxidase (PPO) $A 1 a, A 1 b, D 1 a$ and $D 1 b$ alleles was retrieved from National Centre for Biotechnology Information (NCBI) database (GenBank accession No. EF070147, EF070148, EF070149 and EF070150, respectively). Sequence alignment was performed using ClustalW (https://embnet.vital-it.ch/software/ClustalW.html) and pictorial representation of aligned sequences was prepared using ESPript3 (http://espript.ibcp.fr/ESPript/ ESPript/).

\section{Genotyping at PPOA1 and PPOD1 loci}

Genomic DNA from in vitro germinated wheat seedlings was isolated using a rapid method (Kumar et al., 2017). The primers for PPO33 (Forward: 5'CCAGATACACAACTGCTGGC-3', Reverse: 5'TGATCTTGAGGTTCTCGTCG-3'), PPO16 (Forward: 5'-TGCTGACCGACCTTGACTCC-3', Reverse: 5'CTCGTCACCGTCACCCGTAT-3') and PPO29 (Forward: 5'-TGAAGCTGCCGGTCATCTAC-3', Reverse: 5'-AAGTTGCCCATGTCCTCGCC-3') markers were used in this study, as reported earlier ( $\mathrm{He}$ et al., 2007). PCR-based genotyping at PPOA1 locus was done with the PPO33 marker, whereas genotyping at PPOD1 locus involved the use of PP016 marker (for detection of the PPOD1a allele) and PPO29 marker (for detection of the $P P O D 1 b$ allele). For simultaneous detection of PPOD1a and PPOD1b alleles in co-dominant manner, the combination of PPO16 Forward, PPO29 Forward and PPO16 Reverse primers was used. Each reaction mix (total $12 \mu \mathrm{l}$ ) contained $2 \mu \mathrm{l}$ of isolated genomic DNA, 1X PCR buffer [10 mM Tris-Cl (pH 8.3), 50 $\mathrm{mM} \mathrm{KCl}, 1.5 \mathrm{mM} \mathrm{MgCl}_{2}$ and $0.1 \%$ (v/v) Triton X-100], $0.1 \mathrm{mM}$ of dNTP mix, $0.4 \mu \mathrm{M}$ of each primers, $5 \%(\mathrm{v} / \mathrm{v})$ dimethyl sulfoxide (DMSO) and $1 \mathrm{U}$ of Taq DNA polymerase (Xcelris). The polymerase chain reaction was carried out in an automated thermal cycler (Veriti, Applied Biosystems) with initial denaturation at $94{ }^{\circ} \mathrm{C}$ for 4 min followed by 35 cycles of denaturation at $94{ }^{\circ} \mathrm{C}$ for $30 \mathrm{~s}$, annealing at an appropriate temperature for $40 \mathrm{~s}$ and extension at $72{ }^{\circ} \mathrm{C}$ for $1 \mathrm{~min}$ and ended with a final extension at $72{ }^{\circ} \mathrm{C}$ for $10 \mathrm{~min}$ followed by hold at $4{ }^{\circ} \mathrm{C}$ for $2 \mathrm{~min}$. For the PPO33 marker system, annealing temperature was kept at $61{ }^{\circ} \mathrm{C}$, whereas for the PP016, PPO29 and PPO16 Forward + PPO29 Forward + PPO16 Reverse marker system, the annealing temperature was kept at $64{ }^{\circ} \mathrm{C}$. Following PCR, the amplicons were separated through 1 or $1.2 \%(w / v)$ agarose gel electrophoresis in the presence of ethidium bromide and imaged in gel documentation system (Genei, Bangalore).

\section{Analysis of kernel polyphenol oxidase activity}

Polyphenol oxidase activity of wheat kernels was analyzed through the whole kernel assay method, as de- 


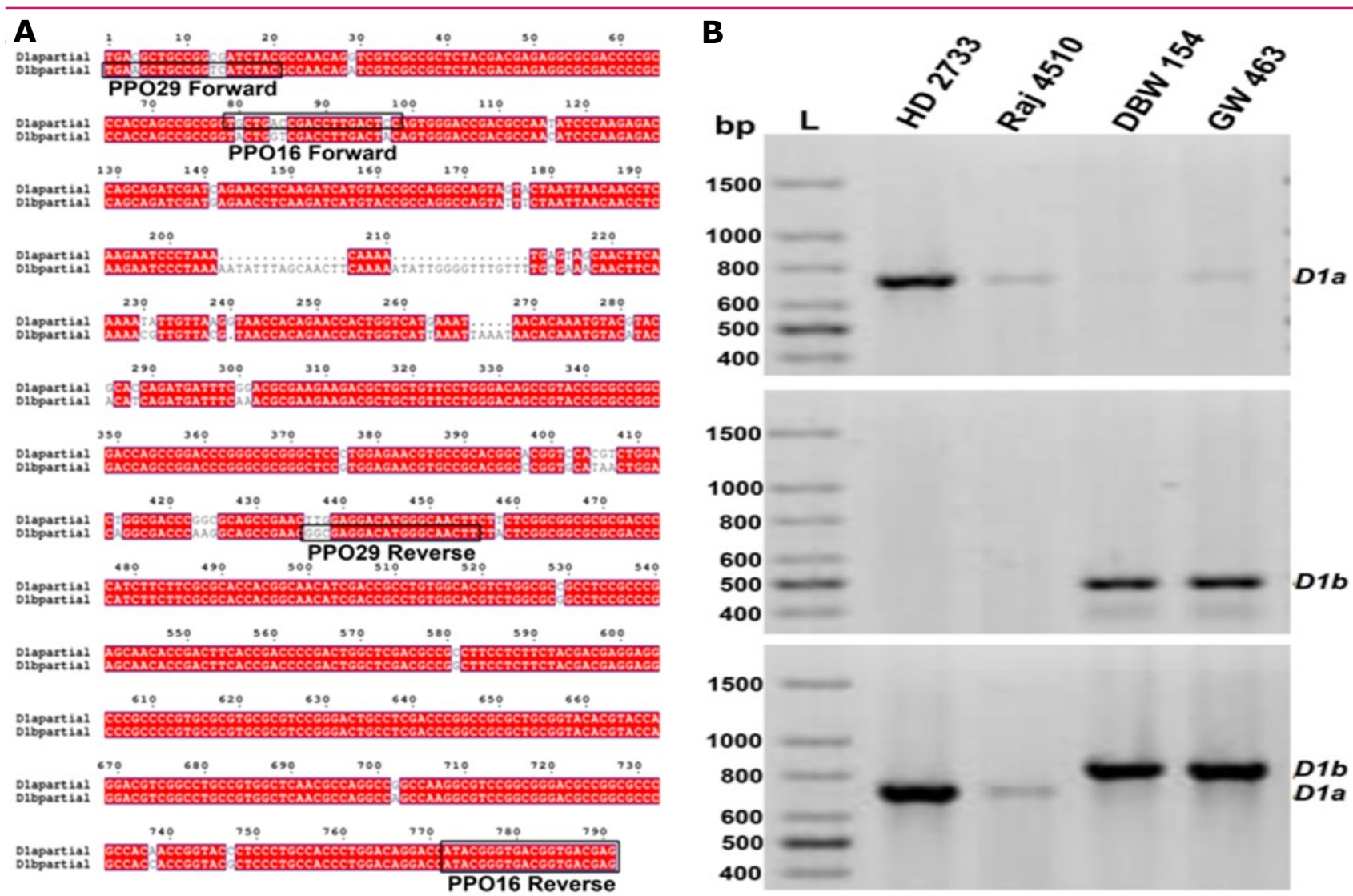

Fig. 1. Development of a strategy for co-dominant screening of alleles at PPOD1 locus. (A) Alignment of partial sequences of PPOD1a and PPOD1b alleles. Region corresponding to PPO16 Forward, PPO16 Reverse, PPO29 Forward and PPO29 Reverse primers are shown inside boxes. (B) Inverse image of ethidium bromide stained $1 \%$ agarose gel showing dominant detection of PPOD1a allele (top gel), dominant detection of PPOD1b allele (middle gel) and co-dominant detection of PPOD1a/PPOD1b allele (bottom gel) in 4 wheat genotypes. $L=100$ bp DNA ladder.

scribed earlier (Singh et al., 2009) with minor modifications. Briefly, 4 randomly selected kernels of each wheat genotype were soaked for $16 \mathrm{~h}$ in doubledistilled water. Afterwards, the kernels were placed on filter paper soaked in $1 \%(\mathrm{w} / \mathrm{v})$ phenol solution and placed in a Petri-dish. After $4 \mathrm{~h}$, the darkening of the kernels was observed and visually scored in a scale of 1 (low) to 4 (high).

\section{RESULTS}

\section{Co-dominant screening of the PPOD1 locus}

Two complementary dominant marker systems (PPO16 and PPO29) have been documented for screening the presence of PPOD1a and PPOD1b alleles in bread wheat (He et al., 2007). In the present study, the partial reference sequences of the PPOD1a and PPOD $1 b$ alleles were aligned in such a way that the sequence alignment could reveal the regions corresponding to the PPO16 and PPO29 marker primers (Fig. 1.A). Interestingly, it was found that the PPO16 Reverse primer could bind perfectly in both PPOD1a and PPOD1b alleles. As PPO16 Forward and PPO29 Forward primers were designed to specifically bind PPOD1a and
PPOD1b alleles, respectively, the use of (PP016 Forward + PPO29 Forward + PPO16 Reverse) primers in a single reaction indicated the possibility of co-dominant screening at PPOD1 locus. Using this primer combination, a $713 \mathrm{bp}$ band specific to $P P O D 1 \mathrm{a}$ allele and an 826 bp band specific to $P P O D 1 b$ allele were expected.

For evaluation of this system, firstly wheat genotypes containing different allelic versions at $P P O D 1$ locus were screened through the PPO16 and PPO29 marker, separately. The wheat genotypes HD 2733 and Raj 4510 produced the PPOD1a allele-specific $713 \mathrm{bp}$ band with the PPO16 marker, whereas the genotypes DBW 154 and GW 463 produced the PPOD1b allelespecific 490 bp band with the PPO29 marker (Fig. 1.B, top and middle gel). Interestingly, the genotype GW 463 was found to generate a faint band of $713 \mathrm{bp}$ with the PP016 marker, which could mislead towards the prediction of the presence of PPOD1a allele in this genotype. Only after obtaining the result with the complementary dominant marker PPO29, the proper allelic version at $P P O D 1$ locus present in this genotype could be confirmed as $P P O D 1 b$. However, the use of PP016 Forward + PPO29 Forward + PPO16 Reverse 
primer combination in a single reaction could easily distinguish between the allelic versions of PPOD1 locus present in the aforementioned 4 wheat genotypes. In the wheat genotypes HD 2733 and Raj 4510, containing the PPOD1a allele, a sharp 713 bp expected band was observed, whereas in the wheat genotypes DBW 154 and GW 463, containing the PPOD1b allele, a sharp 826 bp band was obtained (Fig. 1.B., bottom gel). Thus, the use of PPO16 Forward + PPO29 Forward + PPO16 Reverse primer combination in a single reaction was found to be suitable for co-dominant screening of the PPOD1 locus in wheat.

\section{Testing of heterozygosity at the PPOD1 locus}

The primer combination of PPO16 Forward + PPO29 Forward + PPO16 Reverse was found to be helpful for co-dominant screening for the presence of PPOD1al $P P O D 1 b$ allele in wheat through a single reaction. The co-dominant nature of this primer combination prompted us to check the utility of this system in detecting heterozygosity at $P P O D 1$ locus in wheat. For this purpose, equal amount of genomic DNA from the genotypes HD 2733 (containing the PPOD1a allele), DBW 154 (containing the PPOD1b allele) and GW 463 (containing the PPOD1b allele) were mixed in different combinations to artificially create heterozygosity at the PPOD1 locus. Single PCR with the PP016 Forward + PPO29 Forward + PPO16 Reverse primer combination using the HD 2733 + DBW 154 mixed genomic DNA (representing the PPOD1a/PPOD1b heterozygous genotype) was found to reveal the presence of both the PPOD1a allele-specific $713 \mathrm{bp}$ and PPOD1b allelespecific 826 bp bands (Fig. 2).
In a similar manner, use of this primer combination was found to generate PPOD1a allele-specific $713 \mathrm{bp}$ and PPOD1b allele-specific 826 bp bands, when the HD 2733 + GW 463 mixed genomic DNA (representing the PPOD1a/PPOD1b heterozygous genotype) was used as template for PCR. On the other hand, PCR using DBW 154 + GW 463 mixed genomic DNA (representing the PPOD1b/PPOD1b homozygous genotype) was observed to generate only the $P P O D 1 b$ allele-specific 826 bp band (Fig. 2). Hence, the aforementioned primer combination was found suitable for screening for heterozygosity at the PPOD1 locus, which may arise in the segregating lines of a crossing programme involving parental wheat genotypes carrying different allelic versions at the PPOD1 locus.

\section{Screening at PPOA1 and PPOD1 loci of different wheat genotypes}

Allelic variation at the PPOA1 and PPOD1 loci of 14 wheat genotypes was screened in the present study. For the PPOA1 locus, the co-dominant marker PPO33 (He et al., 2007) was used, whereas, for the PPOD1 locus, the PPO16 Forward + PPO29 Forward + PPO16 Reverse primer combination was used. The PPOA1b allele contained $191 \mathrm{bp}$ insertion in the $1^{\text {st }}$ intron of the gene, which has been explored by the PPO33 marker to differentiate between the PPOA1a and PPOA1b alleles. The PPO33 marker, designed from the conserved sequence flanking this insertion has been reported to generate PPOA1a allele-specific small and PPOA1b allele-specific large amplicons (He et al., 2007). When the 14 wheat genotypes of the present study were subjected to PCR using the PPO33 marker, only 4 geno-

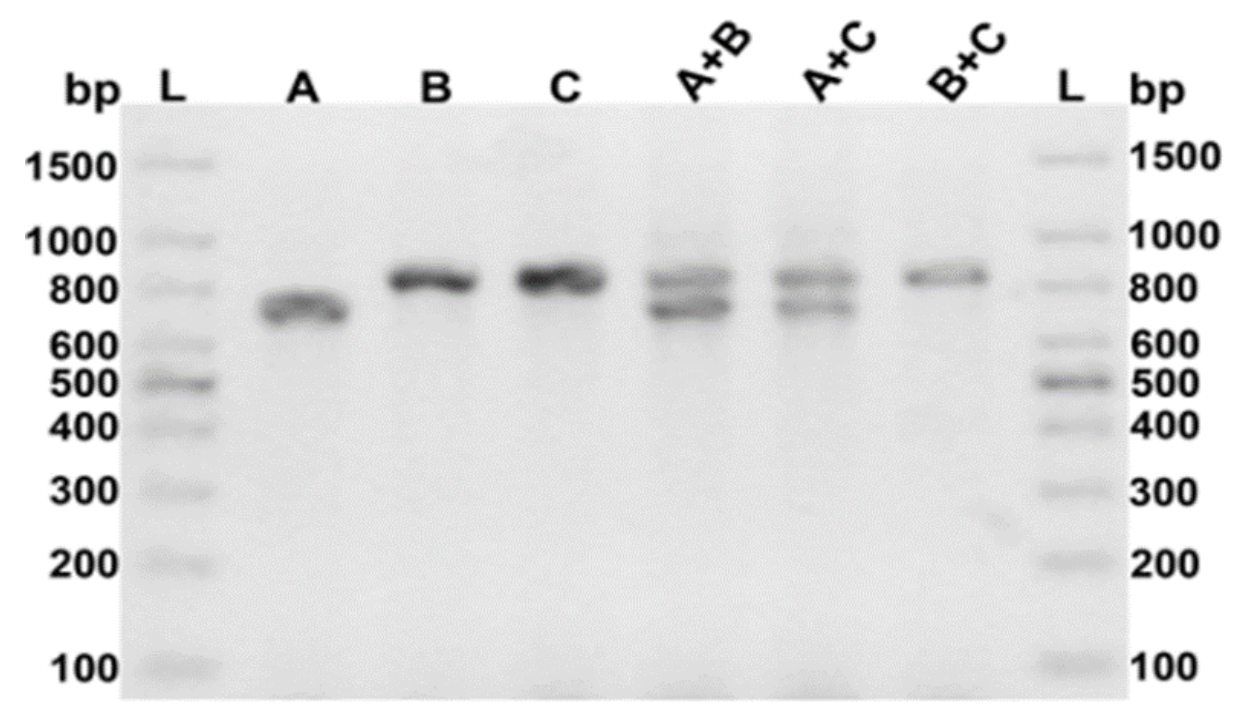

Fig. 2. Inverse image of ethidium bromide stained $1 \%$ agarose gel showing co-dominant detection of PPOD1a and PPOD1b alleles in both homozygous and heterozygous conditions using the (PPO16 Forward + PPO29 Forward + PP016 Reverse) primer combination. $A=H D$ 2733; $B=D B W$ 154; $C=G W 463 ; A+B=$ mixed genomic $D N A$ of $H D$ 2733 and $D B W$ 154; $A+C=$ mixed genomic DNA of HD 2733 and $G W 463 ; B+C=$ mixed genomic DNA of DBW 154 and GW 463; $L=100$ bp DNA ladder. 

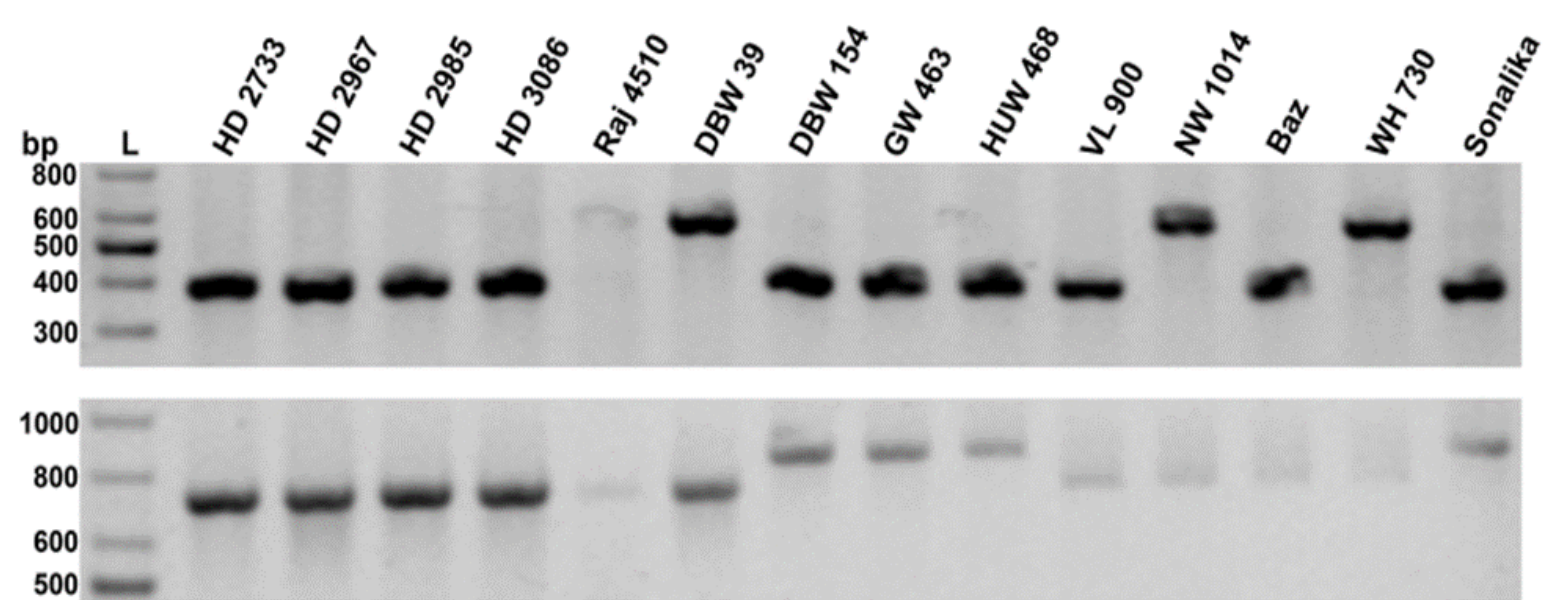

Fig. 3. Inverse image of ethidium bromide stained $1.2 \%$ (top) and $1 \%$ (bottom) agarose gels showing detection of allelic versions at the PPOA1 and PPOD1 loci present in 14 wheat genotypes used in the present study. L = 100 bp DNA ladder.

types (i.e., Raj 4510, DBW 39, NW 1014 and WH 730) were observed to generate the PPOA1b allele-specific 2583 bp band (Fig. 3, top). The other 10 genotypes were found to generate PPOA1a allele-specific $\sim 392 \mathrm{bp}$ band.

The strategy of using the PPO16 Forward + PPO29 Forward + PPO16 Reverse primer combination explored to analyse the allelic variation present at the $P P O D 1$ locus of the 14 wheat genotypes revealed that the PPOD1b allele-specific $\sim 826 \mathrm{bp}$ band was present in only 4 genotypes i.e., DBW 154, GW 463, HUW 468 and Sonalika. The other 10 genotypes were found to generate $P P O D 1$ a allele-specific $\sim 713$ bp band.

Trait-marker relation for polyphenol oxidase (PPO) activity in wheat genotypes

The PPOA1b and PPOD1a alleles at PPOA1 and $P P O D 1$ loci have been documented to be associated with low polyphenol oxidase activity in wheat kernels (Sun et al., 2005; He et al., 2007). In the present study, genotyping of the 14 wheat lines revealed the utility of molecular markers in assessing kernel PPO activity, through phenol test followed by visible scoring (Fig. 4). As summarized in Table 1, presence of the low $P P O$ allelic combination (PPOA1b + PPOD1a) was found in 4 genotypes (i.e., Raj 4510, DBW 39, NW 1014 and WH 730). All of these genotypes, except the genotype WH 730 were observed to possess low kernel polyphenol oxidase activity (Fig. 4). The high PPO allelic combination (PPOA1a + PPOD1b) was present in 3 genotypes (i.e., DBW 154, GW 463 and HUW 468), all with high kernel PPO activity (Fig. 4). Presence of the high PPO allele (PPOA1a) at the PPOA1 locus was found to be associated with high kernel activity in all of the tested wheat genotypes, except one (i.e., VL 900). Thus, the screening of wheat genotypes at PPOA1 and $P P O D 1$ loci using the previously reported PPO33 mark- er and the presently developed primer combination strategy, respectively, was observed to be highly informative about the kernel PPO activity of the genotypes.

\section{DISCUSSION}

In the present study, we studied the kernel PPO activity in wheat genotypes using phenol test and molecular markers. Kernel PPO activity has been found to be an important undesirable feature in wheat-based products, particularly noodles (reviewed in Morris, 2018). Kernel PPO activity in Indian wheat genotypes have been estimated, where genotypes belonging to North Eastern Plain Zone (NEPZ) and North Western Plain Zone (NWPZ) have been identified to have lower kernel PPO activity, making them suitable for use in producing better wheat-based products (Salaria et al., 2018). In our study, for the screening of the allelic variation at PPOD1 locus, 2 complementary dominant markers (PPO16 and PPO29) have been reported (He et al., 2007). Notably, the possibility of erroneous allelic prediction at PPOD1 locus using dominant markers has already been reported (Nilthong et al., 2013). Furthermore, the capability of a co-dominant marker system to distinguish between homozygous and heterozygous segregants offers an added advantage in marker assisted breeding (MAB) programmes. Hence, in this study, we tried to optimize co-dominant screening of the PPOD1 locus in wheat. On the basis of sequence alignment, we developed a co-dominant marker system, where PPO16 Forward, PPO29 Forward and PP016 Reverse primers are used together. Using this strategy, we were able to amplify the PPOD1a allelespecific $\sim 713$ bp band and the PPOD $1 b$ allele-specific 2826 bp band in different wheat genotypes in a codominant manner (Fig. 2, 3). Furthermore, the suitabil- 
A

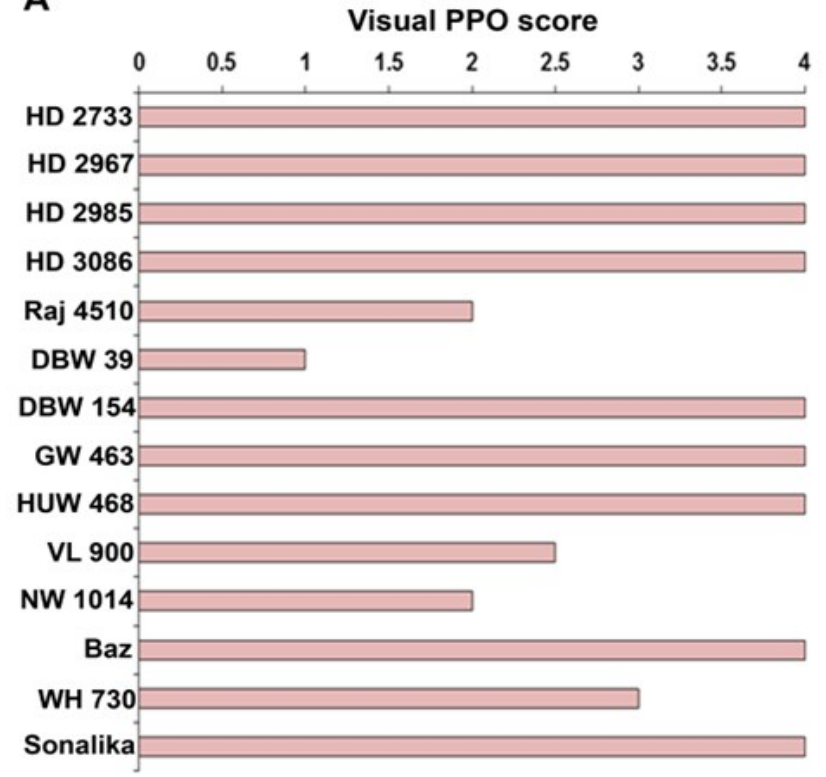

B

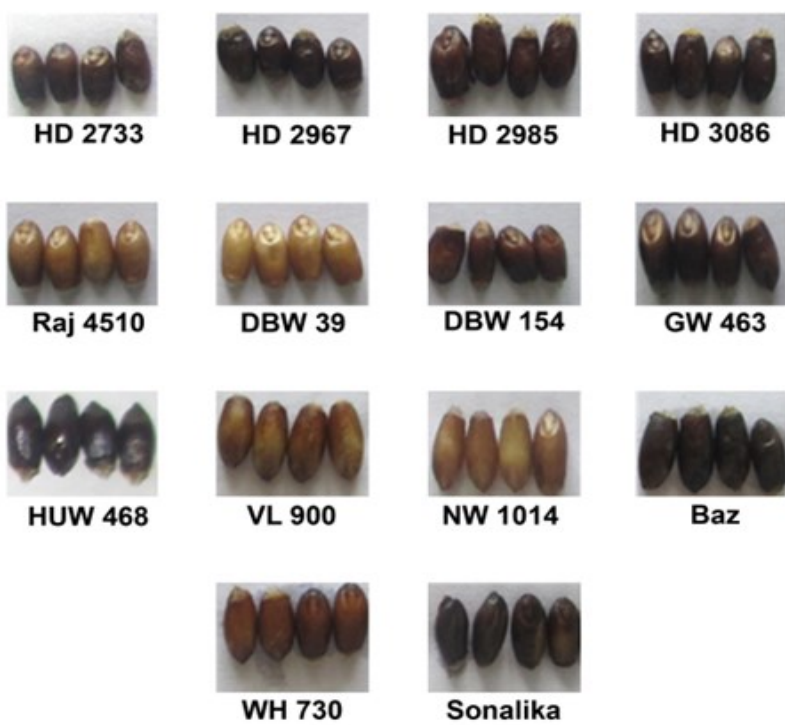

Fig. 4. Kernel polyphenol oxidase activity of 14 wheat genotypes used in the present study. (A) Bar diagram, showing visual score (1 to 4 scale) of kernels from different wheat genotypes after phenol test. (B) Images of enzymatic browning of kernels from different wheat genotypes after phenol test.

Table 1. Allelic status at PPOA1 and PPOD1 loci along with kernel PPO activity in 14 wheat genotypes.

\begin{tabular}{|c|c|c|c|c|}
\hline SI. No. & Genotype & PPOA1 allele & PPOD1 allele & Visual PPO score \\
\hline 1 & HD 2733 & A1a & $\underline{\text { D1a }}$ & 4 \\
\hline 2 & HD 2967 & A1a & $\underline{\text { D1a }}$ & 4 \\
\hline 3 & HD 2985 & A1a & $\underline{\text { D1a }}$ & 4 \\
\hline 4 & HD 3086 & A1a & $\underline{D 1 a}$ & 4 \\
\hline 5 & Raj 4510 & $\underline{A 1 b}$ & $\underline{D 1 a}$ & 2 \\
\hline 6 & DBW 39 & $\underline{A 1 b}$ & $\underline{\text { D1a }}$ & 1 \\
\hline 7 & DBW 154 & A1a & $D 1 b$ & 4 \\
\hline 8 & GW 463 & A1a & $D 1 b$ & 4 \\
\hline 9 & HUW 468 & A1a & $D 1 b$ & 4 \\
\hline 10 & VL 900 & A1a & $\underline{D 1 a}$ & 2.5 \\
\hline 11 & NW 1014 & $\underline{A 1 b}$ & $\underline{D 1 a}$ & 2 \\
\hline 12 & $\mathrm{Baz}$ & A1a & $\underline{D 1 a}$ & 4 \\
\hline 13 & WH 730 & $\underline{A 1 b}$ & $\underline{D 1 a}$ & 3 \\
\hline 14 & Sonalika & A1a & $D 1 b$ & 4 \\
\hline
\end{tabular}

*Alleles reported to be associated with low kernel PPO activity are marker by bold underline

ity of this system in differentiating homozygosity and heterozygosity at the PPOD1 locus was also tested and found appropriate (Fig. 2). Hence, we advocate the utility of this system as an important component in marker-assisted wheat breeding programmes targeting the development of wheat lines with low kernel PPO activity.

The PPOA $1 \mathrm{~b}$ allele has been documented to be associ- ated with low kernel PPO activity (Sun et al., 2005; He et al., 2007). In corroboration to that, all except one of the wheat genotypes carrying the PPOA $1 b$ allele was found to have low kernel PPO activity (Fig. 4, Table 1). In a similar manner, all except one of the 14 wheat genotypes carrying the PPOA1a high PPO activity allele were observed to have high kernel PPO activity, irrespective of the allelic status at the PPOD1 locus 
(Fig. 4, Table 1).

The PPOD1a allele has been documented to be associated with low kernel PPO activity (He et al., 2007). In the present study, all except one genotypes (WH 730) carrying PPOD1a allele, with PPOA1b allele (the low $P P O$ allele at $P P O A 1$ locus) were observed to have low kernel PPO activity (Table 1). However, presence of the PPOA1a allele (the high PPO allele at PPOA1 locus) was found to mask the effect of PPOD1a allele, as all the genotypes, except one (VL 900) carrying the PPOD1a allele with the PPOA1a allele were found to have high kernel PPO activity (Table 1). This observation is supported by previous studies (Raman et al., 2007; Beecher and Skinner, 2011; Martin et al., 2011; Nilthong et al., 2013), where major role of PPOA1 locus in governing kernel PPO activity in wheat has been documented. Moreover, a new gene family (PPOA2, PPOB2 and PPOD2) located on chromosome 2 homeologoues (chromosome 2A, 2B and 2D) has been documented in recent past (Beecher and Skinner, 2011; Beecher et al., 2012), where PPOA2 and $P P O D 2$ along with $P P O A 1$ and $P P O D 1$ loci have been proposed as major regulators of kernel PPO activity in wheat. Furthermore, involvement of several other loci in governing kernel PPO activity has been proposed in a recent past (Zhai et al., 2016; Sadeque et al., 2018; Zhai et al., 2020). Examination of the allelic forms present at PPOA2, PPOD2 and other loci in the wheat genotypes $\mathrm{WH} 730$ and VL900 will be helpful for further exploration of the genetics behind kernel PPO activity in wheat.

\section{Conclusion}

Molecular marker assisted selection (MAS) at PPOA1 and PPOD1 loci can efficiently evaluate the kernel PPO activity in wheat in a rapid, robust and reliable manner. Our strategy of using 3 primers in a single PCR for screening allelic status at $P P O D 1$ locus in co-dominant manner will help the wheat breeders in MAS of wheat genotypes in their breeding programme.

\section{ACKNOWLEDGEMENTS}

The authors thank Bihar Agricultural University, Sabour, Bhagalpur for providing research fund in terms of project grant (Project code: BAU/SNP/Cl/ Rabi/2017-4). AP thanks Bihar Agricultural University for providing financial support in terms of scholarship. The authors acknowledge the help and support provided by Dr. Nitish De, Dr. Chandan Roy and Mr. Sudhir Kumar of Bihar Agricultural University. We also thank Dr. P.K. Singh for his encouragements. This article bears BAU COMMUNICATION NO. $458 / 2018$

\section{Conflict of interest}

The authors declare that they have no conflict of interest.

\section{REFERENCES}

1. Anderson, J.A. \& Morris, C.F. (2001). An improved wholeseed assay for screening wheat germplasm for polyphenol oxidase activity. Crop Sci., 41(6), 1697-1705. https:// doi.org/10.2135/cropsci2001.1697

2. Anderson, J.V., Fuerst, E.P., Hurkman, W.J., Vensel, W.H. \& Morris, C.F. (2006). Biochemical and genetic characterization of wheat (Triticum spp.) seed polyphenol oxidases. J. Cereal Sci., 44(3), 353-367. https://doi.org/1 0.1016/j.jcs.2006.06.008

3. Baik, B.K., Czuchajowska, Z. \& Pomeranz, Y. (1994) Comparison of polyphenol oxidase activities in wheats and flours from Australian and US cultivars. J. Cereal Sci., 19(3), 291-296. https://doi.org/10.1006/jcrs.1994.1036

4. Baik, B.K., Czuchajowska, Z. \& Pomeranz, Y. (1995). Discoloration of dough for oriental noodles. Cereal Chem., 72(2), 198-205. http://online.cerealsgrains.org/publicati ons/cc/backissues/1995/Documents/72 198.pdf

5. Beecher, B.S. \& Skinner, D. (2011). Molecular cloning and expression analysis of multiple polyphenol oxidase genes in developing wheat (Triticum aestivum) kernels. J. Cereal Sci., 53(3), 371-378. https://doi.org/10.1016/j.jcs.2011.0 1.015

6. Beecher, B.S., Carter, A.H. \& See, D.R. (2012). Genetic mapping of a new family of seed-expressed polyphenol oxidase genes in wheat (Triticum aestivum L.). Theor. Appl. Genet., 124, 1463-1473. https://doi.org/10.1007/ s00122-012-1801-2

7. Chang, C., Zhang, H.P., Xu, J., You, M.S., Li, B.Y. \& Liu, G.T. (2007). Variation in two PPO genes associated with polyphenol oxidase activity in seeds of common wheat. Euphytica., 154, 181-193. https://doi.org/10.1007/s10681006-9285-2

8. Fuerst, E.P., Anderson, J.V. \& Morris, C.F. (2006). Delineating the role of polyphenol oxidase in the darkening of alkaline wheat noodles. J. Agric. Food Chem. 54(6), 2378 -2384. https://doi.org/10.1021/jf0526386

9. Gao, Z.J., Han, X.H. \& Xiao, X.G. (2009). Purification and characterisation of polyphenol oxidase from red Swiss chard (Beta vulgaris subspecies cicla) leaves. Food Chem., 117(2), 342-348. https://doi.org/10.1016/j.foo dchem.2009.04.013

10. Hatcher, D.W. \& Kruger, J.E. (1993). Distribution of polyphenol oxidase in flour millstreams of Canadian common wheat classes milled to three extraction rates. Cereal Chem., 70, 51-55. http://online.cerealsgrains.org/public ations/cc/backissues/1993/Documents/70 51.pdf

11. He, X.Y., He, Z.H., Zhang, L.P., Sun, D.J., Morris, C.F., Fuerst, E.P. \& Xia, X.C. (2007). Allelic variation of polyphenol oxidase (PPO) genes located on chromosomes $2 \mathrm{~A}$ and $2 \mathrm{D}$ and development of functional markers for the PPO genes in common wheat. Theor. Appl. Genet., 115, 47-58. https://doi.org/10.1007/s00122-007-0539-8

12. Jime'nez, M. \& Dubcovsky, J. (1999). Chromosome location of genes affecting polyphenol oxidase activity in seeds of common and durum wheat. Plant Breed., 118(5), 
395-398.https://doi.org/10.1046/j.1439-0523.1999.0039 3. $x$

13. Jukanti, A.K., Bruckner, P.L. \& Fisher, A.M. (2004). Evaluation of wheat polyphenol oxidase genes. Cereal Chem., 81(4), 481-485. https://doi.org/10.1094/CCHEM.2004.81. 4.481

14. Kumar, V., Kumar, P. \& Chattopadhyay, T. (2017). A rapid and reproducible method for isolating genomic DNA from a few crop plants suitable for polymerase chain reactionbased genotyping. J. Appl. Nat. Sci. 9(2), 1119-1122. https://doi.org/10.31018/jans.v9i2.1332

15. Mares, D.J. \& Campbell, A.W. (2001). Mapping components of flour and noodle colour in Australian wheat. Aust. J. Agric. Res. 52(11/12), 1297-1309. https://doi.or $\mathrm{g} / 10.1071 /$ AR01048

16. Martin, J.M., Berg, J.E., Hofer, P., Kephart, K.D., Nash, D. \& Bruckner, P.L. (2011). Allelic variation of polyphenol oxidase genes impacts on Chinese raw noodle color. J. Cereal Sci., 54(3), 387-394. https://doi.org/10.1016/ j.jcs.2011.08.003

17. Mayer, A.M. \& Harel, E. (1979). Polyphenol oxidases in plants. Phytochemistry, 18(2), 193-215. https://doi.or g/10.1016/0031-9422(79)80057-6

18. Morris, C.F. (2018) Determinants of wheat noodle color. J. Sci. Food Agric., 98(14), 5171-5180. https://doi.org/10.1 002/jsfa. 9134

19. Nilthong, S., Graybosch, R.A. \& Baenziger, P.S. (2013). Enzyme activity in wheat breeding lines derived from matings of low polyphenol oxidase parents. Euphytica. 190, 65-73. https://doi.org/10.1007/s10681-012-0777-y

20. Park, W.J., Shelton, D.R., Peterson, C.J., Martin, T.J., Kachman, S.D. \& Wehling, R.L. (1997). Variation in polyphenol oxidase activity and quality characteristics among hard white wheat and hard red winter wheat samples. Cereal Chem., 74(1), 7-11. https://doi.org/10.1094/ CCHEM.1997.74.1.7

21. Raman, R., Raman, H. \& Martin, P. (2007). Functional gene markers for polyphenol oxidase locus in bread wheat (Triticum aestivum L.). Mol. Breed., 19, 315-328. https://doi.org/10.1007/s11032-006-9064-8

22. Rani, K.U., Prasada-Rao, U.J.S., Leelavathi, K. \& Haridas -Rao, P. (2001). Distribution of enzymes in wheat flour mill streams. J. Cereal Sci., 34(3), 233-242. https:// doi.org/10.1006/jcrs.2000.0393

23. Sadeque, A., Turner, M., Khan, A., Ahmed, N. \& Adhikari, K. (2018) Identification of quantitative trait loci markers using diversity arrays technology for polyphenol oxidase activity in hexaploid wheat, New Zeal. J. Crop Hort. 46(3), 243-253, https://doi.org/10.1080/01140671.2017.1397 705

24. Salaria, N., Kukreja, S., Kaur, S. \& Goutam, U. (2018) Variable polyphenol oxidase (PPO) activity indicates grain quality in bread wheat (Triticum aestivum L.). Ind. J. Plant Physiol. 23,516-520. https://doi.org/10.1007/s40502-0180379-3

25. Singh, R., Goutam, U., Gupta, R.K., Pandey, G.C., Shoran, J. \& Tiwari, R. (2009). Allelic variations of functional markers for polyphenol oxidase (PPO) genes in Indian bread wheat (Triticum aestivum L.) cultivars. J. Genet., 3, 325-329. https://doi.org/10.1007/s12041-009-0047-5

26. Stevenson, L., Phillips, F., O'sullivan, K. \& Walton, J. (2012). Wheat bran, its composition and benefits to health, a European perspective. Int. J. Food Sci. Nutr., 63 (8), 1001-1013. https://doi.org/10.3109/09637486.2012.6 87366

27. Sun, D.J., He, Z.H., Xia, X.C., Zhang, L.P., Morris, C.F., Appels, R., Ma, W.J. \& Wang, H. (2005). A novel STS marker for polyphenol oxidase activity in bread wheat. Mol. Breed., 16, 209-218. https://doi.org/10.1007/s11032005-6618-0

28. Wang, X.B., Ma, C.X., Si, H.Q., Qiao, Y.Q., Chang, C., He, X.F. \& Xia, Y.X. (2009). Gene markers for grain polyphenol oxidase activity in common wheat. Mol. Breed., 23, 163-170. https://doi.org/10.1007/s11032-008-9223-1

29. Zhai, S., He, Z., Wen, W., Jin, H., Liu, J., Zhang, Y., Liu, Z. \& Xia, X. (2016) Genome-wide linkage mapping of flour color-related traits and polyphenol oxidase activity in common wheat. Theor. Appl. Genet., 129, 377-394. https:// doi.org/10.1007/s00122-015-2634-6

30. Zhai, S., He, Z., Wen, W., Liu, J., Jin, H., Yan, J., Zhang, Y., Zhang, P., Wan, Y. \& Xia, X. (2020). Genetic architecture of polyphenol oxidase activity in wheat flour by genome-wide association study. Crop Sci., 60(3), 12811293. https://doi.org/10.1002/csc2.20038 